\title{
eLS
}

WILEY-

BLACKWELL

\section{Cryptic species and their evolutionary significance}

\begin{tabular}{|c|c|}
\hline Journal: & eLS \\
\hline Manuscript ID & Draft \\
\hline Wiley - Manuscript type: & Standard Article \\
\hline Date Submitted by the Author: & $\mathrm{n} / \mathrm{a}$ \\
\hline \multirow[t]{2}{*}{ Complete List of Authors: } & $\begin{array}{l}\text { Struck, Torsten; University of Oslo, Natural History Museum } \\
\text { Cerca, Jose; University of Oslo, Natural History Museum }\end{array}$ \\
\hline & $\begin{array}{l}\text { Cryptic species are detected at an ever-increasing rate, mainly due to the } \\
\text { application of molecular data. While the impact of this hidden diversity on } \\
\text { macro-ecology and conversation biology is widely recognized, its } \\
\text { evolutionary significance is rarely. In recent years it became apparent that } \\
\text { definitions of cryptic species are too ambiguous to allow the differentiation } \\
\text { between natural phenomena from human-made artefacts. Hence, recently } \\
\text { a unifying conceptual framework has been proposed highlighting the } \\
\text { necessity to test the degree of reduced phenotypic disparity in cryptic } \\
\text { species. Within this reduced disparity also lies the evolutionary } \\
\text { significance, as cryptic species can be regarded as the opposite of adaptive } \\
\text { radiations. Specifically, studies on evolutionary stasis can substantially } \\
\text { benefit from including these by addressing both patterns of reduced } \\
\text { disparity and processes resulting in the lack of phenotypic evolution. } \\
\text { Additionally, this will allow connecting macro-evolutionary and } \\
\text { paleontological studies with micro-evolutionary investigations of genotype- } \\
\text { phenotype linkage. }\end{array}$ \\
\hline
\end{tabular}

\section{SCHOLARONE}




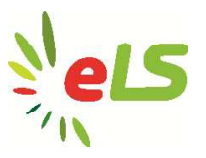

eLS

\section{Cryptic species and their evolutionary significance}

3 A28292

4 Torsten H. Struck, Natural History Museum, University of Oslo, 0318 Oslo,

5 Norway

6 José Cerca, Natural History Museum, University of Oslo, 0318 Oslo, Norway

$7 \quad$ Advanced article

8 Abstract:

9 Cryptic species are detected at an ever-increasing rate, mainly due to the application of

Key words:

Taxonomy; Speciation; Recent divergence; Parallelism; Convergence; Stasis;

Paleontology; Morphology; Phenotype; Adaptive radiations

Key Concepts:

- Cryptic species are phenotypically highly similar species.

- Species complexes resulting from taxonomic artefacts should not be considered as cryptic species.

- To identify cryptic species, first one should establish species boundaries and only then study processes resulting in phenotypic similarity.

- Cryptic species could result from recent speciation, parallelism, convergence or stasis. 


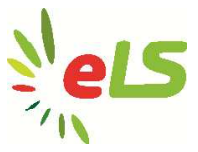

- Evolution of cryptic species through stasis holds the potential to enlighten us about deceleration of phenotypic evolution.

- Cryptic species (shallow morphological differences, pronounced genetic divergence) could be considered as the opposite of adaptive radiation (pronounced morphological differences, shallow genetic divergence).

\section{Introduction:}

Taxonomy, the discipline dealing with the delimitation of biological units, remains one of the most contentious and laborious disciplines in biology, yet it represents a fundamental step before understanding underlying evolutionary processes. Interestingly, taxonomical considerations already had a strong impact on Darwin's line of argument concerning evolution (Darwin, 1859). He had already pointed out the problem of delineating species properly, and that the progress from populations to species is a contiguous scale with respect to the variability that can be observed at different levels, rendering it difficult to delineate species boundaries with certainty. The debate about this problem is still vibrant today and the debate about species concepts and how to apply them is in full fledge (for details see DOI: 10.1002/9780470015902.a0001744.pub2). In the last decades the discussion about cryptic species has been added to this debate, highlighting the necessity to delimitate independently evolving units to understand evolutionary processes.

The concept of cryptic species has been applied as early as 1718 by the English clergyman William Derham focusing on the avian genus Phylloscopus (Winker, 2005) and Mayr (1963) coined the term sibling species for cases, where the species are either sister or very closely related to each other. However, the detection of cryptic species really took off with the advent of employing sequence data in delineating species boundaries on a much broader scale since the early 1990's (Struck et al., 2018b; see DOI: 10.1002/9780470015902.a0003608.pub2 on barcoding). Since then, cryptic species have been detected at an ever-increasing pace and across all habitats on Earth and the entire tree of life including fungi, algae, plants, protists, invertebrates, but also primates, amphibia, reptiles and crustaceans (e.g., Bickford et al., 2007; Pfenninger and Schwenk, 2007; Perez-Ponce de Leon and Poulin, 2016; Hawksworth and Lücking, 2017). Accordingly, cryptic species seem to represent an overlooked, yet substantial part of biodiversity with far-reaching implications for ecological research such as diversity estimates, pest control, fisheries management and conservation efforts as well as research in model systems (Bickford et al., 2007; Caputi et al., 2007; Bernardo, 2011; Pante et al., 2015; Fišer et al., 2018). However, like with any new emerging concept in biology, the term cryptic species is often applied with very different meanings and hence has different implications. This, among others, affects the general conclusions, which can be drawn concerning cryptic species. Due to the different meanings the conducted metaanalyses usually compare apples with oranges (Struck et al., 2018b; Struck et al., 2018a). Therefore, it became noticeable that a unifying theoretical framework is needed for studying cryptic species, which then would also allow for drawing more general and solid conclusions about the ecological and evolutionary significance of cryptic species. Only such conclusions are then able to provide meaningful contributions to, for example, conversation management or health issues stemming from cryptic species. 


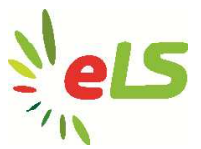

\section{Towards a unifying conceptual framework for cryptic species}

2

3

4

5

6

7

8

9

10

11

12

13

14

15

16

17

18

19

20

21

22

23

24

25

26

27

28

29

30

31

32

33

34

35

36

37

38

39

40

41

42

43

44

45

46

The first attempt towards a unifying conceptual framework of cryptic species was accomplished by Bickford et al. (2007). They defined cryptic species as "two or more distinct species that are erroneously classified (and hidden) under one species name". This broad definition was quickly applied in several studies and became the most commonly used one, as it is easily applicable due to its tight link to the taxonomic history of the species. That is, it strictly requires that the species complex is formally described as a single species before. However, this also entails a caveat associated with different schools of taxonomic practice (DOI: 10.1038/npg.els.0003093). For instance, in taxonomy some schools favour splitting species even in cases of only little support, while others would rather lump these together as a single species (the splitter vs. lumper debate). Hence, some groups would be more prone to have cryptic species only due to different taxonomic practices. For example, the newly described Marphysa aegypti (Annelida) from Egyptian waters was previously recorded as $M$. sanguinea (Elgetany et al., 2018). Even though both species are morphologically substantially different from each other, they could be called cryptic species or at least pseudo-cryptic species, while in truth the previous records suggest only sloppy taxonomic practices. On the other hand, molecular data supported the traditional assignment of species within the genus Polygordius (Annelida) based on geographic regions (Ramey-Balcl et al., 2018), despite the indistinguishable adult morphology of $P$. lacteus from $P$. neapolitanus and $P$. jouinae from $P$. triestinus. Nonetheless, both species pairs could not be considered cryptic species given the provided definition by Bickford et al. (2007). Moreover, others pointed out that recent definitions of cryptic species are often linked to and depend on the applied species concept (Pante et al., 2015; Sukumaran and Knowles, 2017; Fišer et al., 2018; Heethoff, 2018). Given these non-biological aspects, several studies introduced slightly different concepts such as pseudo-cryptic, hyper- or mega-cryptic species (e.g., Adams et al., 2014; Cornils and Held, 2014; Nygren et al., 2018).

Hence, in recent years there has been an increased debate again what constitutes a cryptic species. Ultimately, Korshunova et al. (2017) argued "to avoid the terms "cryptic"/"pseudocryptic" species as a reference to a "natural phenomenon" because it is obscuring multilevel character diversity within a complicated taxonomy-dependent framework" and instead "to use the term "cryptic species" only for a temporary formalization of the problems with delineation of the species from the same geographic region, when those species demonstrate significant molecular phylogenetic differences, but are hardly distinguished morphologically, ethologically, etc.". A recent literature survey found that only $14 \%$ of the studies actually provided or applied an explicit definition of cryptic species adding to the problem of uncertainty in the assignment of cryptic species (Struck et al., 2018b). Of these, all were explicitly or implicitly taxonomybased like the Bickford et al. (2007) definition. Additional criteria such as usage of molecular data, sympatric occurrence, reproductive isolation, reduced gene flow, or no morphological differences were included in the definitions. Moreover, another problem of assigning cryptic species based on these definitions is that the species delineation process is intermingled with the assignment of the term "cryptic". As a consequence, all problems associated with species delineation (DOI: 10.1002/9780470015902.a0003456.pub2) also automatically apply to the assignment. In summary, the assignment of a species as cryptic species depends on many non- 


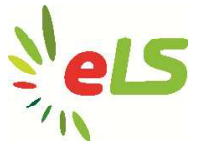

biological factors such as the applied definition and species concept as well as the taxonomic tradition resulting in much uncertainty about what constitutes a cryptic species and if it is a natural phenomenon or only a human-made artefact. This problem has dire consequences for meta-analyses investigating the impact of cryptic species in biodiversity studies (e.g., Perez-Ponce de Leon and Poulin, 2016; Poulin and Pérez-Ponce de León, 2017), and for the understanding of biological processes.

Two general assumptions, which were at least implicitly applied in the practical procedures, were evident from the literature (Struck et al., 2018b). First, given the species concept cryptic species were generally thought to be "true" species and, second, these species were so similar in the taxonomically relevant phenotypic characters that they were not or hardly distinguishable from each other. Therefore, Struck et al. (2018b; 2018a) provided a new definition for cryptic species reflecting these two assumptions. The process of assigning cryptic species was separated into two clearly separated steps in contrast to the previous attempts. At the first step, it has to be established that the species are truly species given the applied species concept. In Figure 1 the white circles indicate cases, which would not be considered species and accordingly also not cryptic species. Hence, this first step is not different from any other species delineation process, independent if these entities are cryptic or not, but it has the advantage that the pitfalls associated with this process are confined to the proper step and are not carried over to the next step. The second step consists in showing that the species are phenotypically more similar to each other than one would expect given the time that has passed since their last common ancestor (or the level of genetic divergence as a proxy for time). These species should hence be called cryptic species only if this level of phenotypic disparity is significantly lower than expected. The red circles in Figure 1 represent such cases, while the orange and yellow circles do not. This second step is the crucial step in the framework with respect to cryptic species as here the actual assignment occurs. The definition is property-based and independent of the taxonomic history of the species at hand. Specifically, it does not matter if the species have been described as only one before or not. Studies across taxa, habitats, life strategies and so forth can be based on comparable categories like similar applied species concepts, levels of disparity, or genetic divergence instead of taking cryptic species at face value allowing more robust conclusions about the impact of cryptic species.

\section{Evolutionary significance of cryptic species}

Understanding the tempo and mode of speciation, and the drivers of phenotypic diversification are major objectives in biology (Rabosky and Adams, 2012). Therefore, identifying and quantifying contrasts between phenotypic disparity and genetic divergence has become popular in recent decades. Cases such adaptive radiations where morphological disparity outpaces genetic divergence received considerable attention (DOI: $10.1038 / \mathrm{npg}$.els.0001782). Adaptive radiations generally rely on open ecological opportunities like appearance of new resources, evolution of key innovations or colonization of new areas followed by specialization to the open niches and speciation (Losos, 2010). Cryptic species as defined by the aforementioned framework can be regarded as the exact opposite to these radiations, being characterized by a strongly reduced phenotypic or at least morphological disparity in comparison to the observed genetic divergence (yellow vs. red circles in Fig. 1). 
If high rates of phenotypic variation have the potential to result in radiation of organisms and occupation of different evolutionary niches - "high evolvability" - then low phenotypic disparity as observed in cryptic species can be seen as a paradox as theory predicts that clades with high evolvability supersede clades with low evolvability (Estes and Arnold, 2007; Rabosky and Adams, 2012). Clades of cryptic species should have a lower "adaptive zone" and occupy less ecological space and, hence, be replaced by clades with a potential to evolve and adapt faster (Rabosky and Adams, 2012). As evolvability is generally considered as a measure of evolutionary success (e.g., Rabosky and Adams, 2012) the question arises why so many cryptic species are observed nowadays, which supposedly lack any phenotypic or at least morphological evolution. Several suggestions have been put forth to explain lack of phenotypic evolution in general and especially considering macro-evolutionary patterns (Futuyma, 2010). These include, among others, genetic and developmental constraints, source populations impeding specialization (meta-population dynamics), repeated bottlenecks decreasing standing genetic variation, large populations, stabilizing selection, ephemeral, stressful or fluctuating environments, evolutionary stable configurations, or niche conservationism (e.g., Maynard Smith, 1983; Eldredge et al., 2005; Futuyma, 2010; Haller and Hendry, 2014; Chomicki and Renner, 2017). However, the lack of phenotypic evolution has received considerably less attention in evolutionary biology than its opposite, adaptive radiations, especially at the micro-evolutionary level, and empirical and experimental evidence for any of the suggestions is low so far. While cryptic species can be ideal systems to inform us on the causes of reduced phenotypic disparity, much needs to be done. First, the evolutionary processes resulting in cryptic species must be identified. Then it can be investigated in how far the different causes mentioned above influenced these processes. Four different processes have been suggested to result in cryptic species: recent divergence, convergence, parallelism and stasis (Swift et al., 2016; Struck et al., 2018b).

\section{Evolutionary Processes I: Recent divergence}

The most common, but unproven assumption is that cryptic species follow recent speciation and that they did not yet have enough time to accumulate substantial phenotypic, especially morphological differences (Knowlton, 1993; Reidenbach et al., 2012). For example (Fig. 2), in the malaria vector Anopheles gambiae (Hexapoda) two forms, the $M$ (now recognized as $A$. coluzzii) and $S$ form, are recognized, which seem to be reproductively isolated (Reidenbach et al., 2012). They are at an early stage after speciation differing in an inversion on chromosome-2, which seems to be associated with their ecological differences (Simard et al., 2009). The $M$ form mainly exploits stable larval habitats with high level of stressors; the S form exploits unpolluted, predator free, ephemeral habitats associated with seasonal rainfall (Reidenbach et al., 2012). Ecological experiments suggest that these forms seem to outcompete each other in their respective environment and respond to predation differently (Diabaté et al., 2008). Hence, in the $\mathrm{M}$ and $\mathrm{S}$ forms other traits than morphology are under selection.

Speciation is not necessarily accompanied by morphological change in the early stages as selection acts largely on physiological, immunological, reproductive or behavioural traits (Bensch et al., 2004; Damm et al., 2010; Derycke et al., 2016). Allopatric speciation while remaining in one particular ecological niche or habitat might lead to the building-up of non-adaptive divergence without morphological change. As a result 


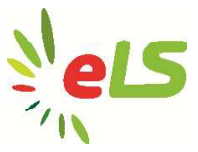

recently diverged species can remain morphologically identical following initial divergence (Korshunova et al., 2017). In these cases of recent divergence cryptic species are nothing special to other species pairs as the speciation event is very recent and generally no or little phenotypic change, particularly in morphology, is expected this shortly after the speciation unless there is a strong selection towards different adaptive optima. Moreover, such cases cannot provide much insight into the supposed lack of phenotypic evolution.

\section{Evolutionary Processes II: Convergence \& Parallelism}

Phenotypic similarity could also stem from convergence or parallelism (Swift et al., 2016; Struck et al., 2018b). In both cases the cryptic species evolved the same phenotypes independently of each other. While for convergence the immediate ancestors of the cryptic species were dissimilar from each other as well as to the extant cryptic species, for parallelism the ancestors were similar to each other, but dissimilar to the extant cryptic species. One example (Fig. 3) comprising both convergence and parallelism occurs in the Mastigias species complex (Scyphozoa) (Swift et al., 2016). Mastigias species occur in both coastal waters including coves and lagoons ('ocean' phenotypes), and in small-bodies of salt water without an open connection to the ocean ('lake' phenotypes). The 'lake' phenotypes evolved both by parallelism and convergence from the 'ocean' phenotypes. In some cases, the 'lake' phenotypes evolved from the same 'ocean' phenotype (parallelism) and in others from different 'ocean' phenotypes (convergence). Similarly, selective pressures from predators, led to parallelism in the Holarctic Enallagma species (Hexapoda) (Stoks et al., 2005). Cases of convergence of cryptic species were also found in the Deep Sea (Vrijenhoek, 2009).

As for the case of recent divergence the evolutionary processes of convergence and parallelism cannot contribute to our understanding of the lack of phenotypic evolution, as phenotypic change occurred. Nonetheless, these cases can help us understand how reduced phenotypic disparity evolves. Importantly, it indicates that reduced phenotypic disparity and lack of phenotypic evolution are not necessarily the same. For convergence and parallelism specifically the question arises what are the driving factors that the cryptic species independently evolved to the same phenotypes? Is it due to intrinsic (e.g., developmental constraints) or extrinsic ones (e.g., extreme environments) confining the available phenotypic landscape to only one solution in the respective situation? Contrary to parallelism, intrinsic factors are expected to be less influential than extrinsic ones in convergence, as evolution starts from more distinct genetic backgrounds. However, maybe even for convergence developmental constraints constrict the available phenotypic landscape more than expected.

\section{Evolutionary Processes III: Stasis}

In contrast to the other three processes, under phenotypic stasis cryptic species retain similar phenotypes for millions of years. The literature survey by Struck et al. (2018b) revealed that stasis in cryptic species may occur at least not substantially less than recent divergence and seems to be an important process in the evolution of cryptic species. A prominent example (Fig. 4) is provided by the Cavernacmella complex (Gastropoda) on the Ogasawara islands (Japan) (Wada et al., 2013). The 'C. minima' phenotype occurs in five clades on the different islands of the archipelago (i.e., 
Mukojima, Chichijima, and Hahajima). These represent cryptic species and are unaltered for over 3 million years. In contrast, within the clade of these cryptic species are also five species, which are morphologically distinct from the ' $C$. minima' phenotype. This indicates that enough time passed to accumulate morphological differences under certain conditions. This release from morphological arrest might indicate the absence of developmental constraints. Similarly, cichlids have demonstrated the potential for burst of morphological evolution and stasis (Seehausen, 2006). Other examples of stasis comprise Stygocapitella (Annelida), Diporiphora (Squamata), Mastigias (Scyphozoa), or Cletocamptus (Crustacea) species complexes (Rocha-Olivares et al., 2001; Smith et al., 2011; Swift et al., 2016; Struck et al., 2017). Stygocapitella is an example of long lasting morphological stasis. Stygocapitella individuals live between sand grains, by the foot of the dune, at a depth of about 0.5-1 meter and can be found on beaches in all major coastlines with the exception of tropical regions (Westheide, 2008). Molecular data suggest the presence of several cryptic species at the different coastlines and even though rigorous morphological reinvestigations discovered minimal phenotypic differences among the deeply divergent clades, these are estimated to have diverged about 300 to 100 million years ago (Struck et al., 2017). As such, the Stygocapitella complex seems to be under morphological stasis with slight morphological differentiations having occurred more than 100 million years ago. It is certainly challenging to address why these species did not change morphologically while closely related ones did, and why no phenotypic differences became fixed in the gene pools just by chance (e.g., due to recurrent bottlenecks).

Hence, cryptic species such as the presented examples are ideal systems to investigate stasis using extant taxa. These systems allow addressing both patterns of reduced phenotypic disparity and the process leading to the absence of phenotypic evolution. The term stasis is most often used in macro-evolutionary and paleontological studies and less in micro-evolutionary ones using extant taxa. In macroevolution and paleontology it became popularized as an argument for punctuated equilibria (DOI: 10.1038/npg.els.0001774), where evidence from fossil timelines lasting for millions of years questioned the power of selection. However, recent efforts have focused on integrating stasis at the micro-evolutionary level (e.g., Hansen and Houle, 2004; Estes and Arnold, 2007). Futuyma (2010) reviewed many of these models including stabilizing selection, lack of genetic diversity, genetic and developmental constraints, ecological niche tracking and niche conservatism. Other potential, non-exclusive explanations for stasis or arrested evolution have been put forward specifically for more specific scenarios or habitats. For example, for the interstitial realm, that is the space between the sand grains in marine sediments, one potential explanation stems from the 'plus ça change, plus c'est la même chose' (The more it changes, the more it is the same) model (Sheldon, 1996). This model regards morphological stasis as a response to widely fluctuating physical conditions, which are stable on geological timescales. This fits the interstitial realm, as this is characterized by wide variations in $\mathrm{pH}$, salinity and moisture at short timescales, but this fluctuating characteristic has remained similar for millions of years ago (e.g., Westheide, 1977). It has also been suggested that the phenotypic landspace of species with limited morphological differentiation such as, e.g., some worms or fungi are too small to allow for change (e.g., Bickford et al., 2007). In contrast, another suggestion is that intraspecific variation of traits is high and allows for 


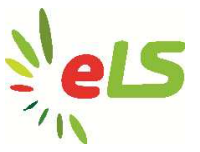

1 coping with broader ranges of ecological differences, while the traits fluctuate around a

2 stable mean (Voje, 2016).

\section{Conclusions}

4 Studying cryptic species has great potential to further our understanding of evolutionary 5 processes as outlined above, but also with respect to research in macroecology and 6 conservation biology (Fig. 5) (Bickford et al., 2007; Bernardo, 2011; Pante et al., 2015; 7 Fišer et al., 2018). However, to accomplish these goals a pre-requisite is that what is a 8 cryptic species can be determined with certainty. It is important to differentiate cryptic 9 species complexes from those complexes arising from taxonomic biases or malpractice. 10 Only the former will be able to inform us on biological processes in evolution and ecology as they reflect true natural properties. A two-step conceptual framework to accomplish this has been provided and hence the theoretical foundation been laid. However, the literature survey by Struck et al. (2018b) also clearly showed that methodological improvement is needed in all aspects to achieve this. Often the phenotype is not studied at all, only a single, uni-parentally inherited genetic marker is used, and/or the results are not set in relation to time or other non-cryptic species to assess if they are really exceptionally different from them. Moreover, biology is transforming into a 'big data' science including among others high-throughput sequencing technologies, which allows us to apply population genomic and phylogenomic methods independent of the study object and hence provide much broader data basis for their conclusions (Fig. 5).

On the other hand, improved delineations of cryptic species will result in and contribute to an improved understanding of the causes of evolutionary processes like convergence, parallelism and stasis (Fig. 5). Again, the applications of genomic and transcriptomic approaches studies on cryptic species can aid in linking genotypic change to phenotypic alterations or lack thereof. Similarly, improved delineations will result in improved biodiversity assessments allowing better modelling of macroecological processes. Moreover, an improved understanding of the evolutionary processes shaping cryptic species allows a better assessment of ecological changes and its evolutionary consequences over space and time. Summarizing the research on cryptic species should address the following questions to contribute to our understanding of the evolution of reduced phenotypic disparity and how this affects the macroecological processes in different habitats:

33 1) Which species complexes are only taxonomic oddities, and which are truly cryptic?

34 2) Which cryptic species are the results of recent speciation, parallelism, convergence or 35 stasis, and how common are they?

36 3) What are the relevant intrinsic and extrinsic factors affecting phenotypic evolution of cryptic species and to what degree do they affect their phenotypic landscape?

38 4) Are there more cryptic species in certain branches of the tree of life, among taxa with certain life histories (e.g., generalists vs. specialists), or in certain habitats?

41 how vulnerable are cryptic species due to these effects? 
2

3

4

5

6

7

8

9

10

11

12

13

14

15

16

17

18

19

20

21

22

23

24

25

26

27

28

29

30

31

32

33

34

35

36

37

38

39

40

41

42

43

44

45

46

47

48

49

50

51

52

53

\section{References:}

Adams M, Raadik TA, Burridge CP, Georges A (2014) Global biodiversity assessment and hyper-cryptic species complexes: More than one species of elephant in the room? Systematic Biology 63(4): 518-533.

Bensch S, Pérez-Tris J, Waldenström J, Hellgren O (2004) Linkage between nuclear and mitochondrial DNA sequences in avian malaria parasites: Multiple cases of cryptic speciation? Evolution 58(7): 1617-1621.

Bernardo J (2011) A critical appraisal of the meaning and diagnosability of cryptic evolutionary diversity, and its implications for conservation in the face of climate change. Climate change, ecology and systematics., Hodkinson TR, Jones MB, Waldren S, Parnell JAN, editors, 380-438. Cambridge, UK: Cambridge University Press.

Bickford D, Lohman DJ, Sodhi NS et al. (2007) Cryptic species as a window on diversity and conservation. Trends in Ecology \& Evolution 22(3): 148-155.

Caputi L, Andreakis N, Mastrototaro F et al. (2007) Cryptic speciation in a model invertebrate chordate. Proceedings of the National Academy of Sciences 104(22): 93649369.

Chomicki G, Renner SS (2017) Partner abundance controls mutualism stability and the pace of morphological change over geologic time. Proceedings of the National Academy of Sciences 114(15): 3951-3956.

Cornils A, Held C (2014) Evidence of cryptic and pseudocryptic speciation in the paracalanus parvus species complex (crustacea, copepoda, calanoida). Frontiers in Zoology 11): 19.

Damm S, Schierwater B, Hadrys H (2010) An integrative approach to species discovery in odonates: From character-based DNA barcoding to ecology. Molecular Ecology 19): 3881-3893.

Darwin C (1859) On the origin of species by means of natural selection: Or the preservation of favoured races in the struggle of life. London: John Murray.

Derycke S, De Meester N, Rigaux A et al. (2016) Coexisting cryptic species of the litoditis marina complex (nematoda) show differential resource use and have distinct microbiomes with high intraspecific variability. Molecular Ecology 25): 2093-2110 Diabaté A, Dabiré RK, Heidenberger K et al. (Diabaté2008 co-authors)(2008) Evidence for divergent selection between the molecular forms of anopheles gambiae: Role of predation. BMC Evolutionary Biology 8(1): 5.

Eldredge N, Thompson JN, Brakefield PM et al. (2005) The dynamics of evolutionary stasis. Paleobiology 31(sp5): 133-145.

Elgetany AH, El-Ghobashy AE, Ghoneim AM, Struck TH (2018) Description of a new species of the genus marphysa (eunicidae), marphysa aegypti sp.N., based on molecular and morphological evidence. Invertebrate Zoology 15(1): 71-84.

Estes S, Arnold SJ (2007) Resolving the paradox of stasis: Models with stabilizing selection explain evolutionary divergence on all timescales. The American Naturalist 169(2): 227-244.

Fišer C, Robinson CT, Malard F (2018) Cryptic species as a window into the paradigm shift of the species concept. Molecular Ecology 27): 613-635.

Futuyma DJ (2010) Evolutionary constraint and ecological consequences. Evolution 64(7): 1865-1884.

Haller BC, Hendry AP (2014) Solving the paradox of stasis: Squashed stabilizing selection and the limits of detection. Evolution 68(2): 483-500.

Hansen TF, Houle D (2004) Evolvability, stabilizing selection, and the problem of stasis. Phenotypic integration: Studying the ecology and evolution of complex phenotypes., Pigliucci M, Preston K, editors, 130-154. Oxford: Oxford University Press. Hawksworth DL, Lücking R (2017) Fungal diversity revisited: 2.2 to 3.8 million species. Microbiology Spectrum 5(4). 


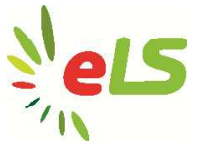

Heethoff M (2018) Cryptic species - conceptual or terminological chaos? A response to struck et al. Trends in Ecology \& Evolution 33(5): 310.

Knowlton N (1993) Sibling species in the sea. Annual Review of Ecology and Systematics 24(1): 189-216.

Korshunova T, Martynov A, Bakken T, Picton B (2017) External diversity is restrained by internal conservatism: New nudibranch mollusc contributes to the cryptic species problem. Zoologica Scripta 46): 683-692.

Losos Jonathan B (2010) Adaptive radiation, ecological opportunity, and evolutionary determinism. The American Naturalist 175(6): 623-639.

Maynard Smith J (1983) The genetics of stasis and punctuation. Annual Review of Genetics 17(1): 11-25.

Mayr E (1963) Animal species and evolution. Cambridge: Belknap Press of Harvard University.

Nygren A, Parapar J, Pons J et al. (2018) A mega-cryptic species complex hidden among one of the most common annelids in the north east atlantic. PLOS ONE 13(6): e0198356. Pante E, Puillandre N, Viricel A et al. (2015) Species are hypotheses: Avoid connectivity assessments based on pillars of sand. Molecular Ecology 24(3): 525-544.

Perez-Ponce de Leon G, Poulin R (2016) Taxonomic distribution of cryptic diversity among metazoans: Not so homogeneous after all. Biology Letters 12): 20160371. Pfenninger M, Schwenk K (2007) Cryptic animal species are homogeneously distributed among taxa and biogeographical regions. BMC Evolutionary Biology 7(1): 121. Poulin R, Pérez-Ponce de León G (2017) Global analysis reveals that cryptic diversity is linked with habitat but not mode of life. Journal of Evolutionary Biology 30(3): 641-649. Rabosky DL, Adams DC (2012) Rates of moprhological evolution are correlated with species richness in salamanders. Evolution 66(6): 1807-1818.

Ramey-Balcı P, Fiege D, Struck TH (2018) Molecular phylogeny, morphology, and distribution of polygordius (polychaeta: Polygordiidae) in the atlantic and mediterranean. Molecular Phylogenetics and Evolution 127): 919-930.

Reidenbach KR, Neafsey DE, Costantini C et al. (2012) Patterns of genomic differentiation between ecologically differentiated $\mathrm{m}$ and $\mathrm{s}$ forms of anopheles gambiae in west and central africa. Genome Biology and Evolution 4(12): 1202-1212.

Rocha-Olivares A, Fleeger JW, Foltz DW (2001) Decoupling of molecular and morphological evolution in deep lineages of a meiobenthic harpacticoid copepod. . Molecular Biology and Evolution 18): 1088-1102.

Seehausen $O$ (2006) African cichlid fish: A model system in adaptive radiation research. Proceedings of the Royal Society B: Biological Sciences 273(1597): 1987-1998. Sheldon PR (1996) Plus ça change - a model for stasis and evolution in different environments. Palaeogeography, Palaeoclimatology, Palaeoecology 127(1): 209-227. Simard F, Ayala D, Kamdem GC et al. (Simard2009 co-authors)(2009) Ecological niche partitioning between anopheles gambiae molecular forms in cameroon: The ecological side of speciation. BMC Ecology 9(1): 17.

Smith KL, Harmon LJ, Shoo LP, Melville J (2011) Evidence of constrained phenotypic evolution in a cryptic species complex of agamid lizards. Evolution 65(4): 976-992. Stoks R, Nystrom JL, May ML, McPeek MA, Benkman C (2005) Parallel evolution in ecological and reproductive traits to produce cryptic damselfly species across the holarctic. Evolution 59(9): 1976-1988.

Struck TH, Feder JL, Bendiksby M et al. (2018a) Cryptic species - more than terminological chaos: A reply to heethoff. Trends in Ecology \& Evolution 33(5): 310-312. Struck TH, Feder JL, Bendiksby M et al. (2018b) Finding evolutionary processes hidden in cryptic species. Trends in Ecology \& Evolution).

Struck TH, Koczula J, Stateczny D, Meyer C, Purschke G (2017) Two new species in the annelid genus stygocapitella (orbiniida, parergodrilidae) with comments on their biogeography. Zootaxa 4286(3): 301-332. 
Sukumaran J, Knowles LL (2017) Multispecies coalescent delimits structure, not species. Proceedings of the National Academy of Sciences 114(7): 1607-1612.

Swift HF, Gómez Daglio L, Dawson MN (2016) Three routes to crypsis: Stasis, convergence, and parallelism in the mastigias species complex (scyphozoa, rhizostomeae). Molecular Phylogenetics and Evolution 99): 103-115. Voje KL (2016) Tempo does not correlate with mode in the fossil record. Evolution 70(12): 2678-2689.

Vrijenhoek RC (2009) Cryptic species, phenotypic plasticity, and complex life histories: Assessing deep-sea faunal diversity with molecular markers. Deep Sea Research Part II: Topical Studies in Oceanography 56(19): 1713-1723. Wada S, Kameda Y, Chiba S (2013) Long-term stasis and short-term divergence in the phenotypes of microsnails on oceanic islands. Molecular Ecology 22(18): 4801-4810. Westheide W (1977) The geographical distribution of interstitial polychaetes. Mikrofauna Meeresboden 61): 287-302.

Westheide W (2008) Polychaetes: Interstitial families. Shrewsbury: Field Studies Council. Winker K (2005) Sibling species were first recognized by william derham (1718). Auk 122): $706-707$.

\section{Further Reading:}

Coyne J, Orr H (2004) Speciation. Sunderland, MA: Sinauer Associates. Winston JE (1999) Describing species - practical taxonomic procedure for biologists. New York: Columbia University Press.

Giere O (2009) Meiobenthology - the microscopic motile fauna of aquatic sediments. Berlin Heidelberg: Springer-Verlag.

Cerca J, Purschke G, Struck TH (2018) Marine connectivity dynamics: clarifying cosmopolitan distributions of marine interstitial invertebrates and the meiofauna paradox. Marine Biology 165:123.

Charlesworth, B., R. Lande, and M. Slatkin. 1982. A Neo-Darwinian Commentary on Macroevolution. Evolution 36: 474-498.

Schwenk K, Wagner GP (2001) Function and the evolution of phenotypic stability: Connecting pattern to process. American Zoologist 41(3): 552-563.

Nygren A (2013) Cryptic polychaete diversity: A review. Zoologica Scripta 43: 172-183. Appeltans W, Ahyong Shane T, Anderson G et al. (2012) The magnitude of global marine species diversity. Current Biology 22(23): 2189-2202.

Karanovic T, Djurakic M, Eberhard SM (2016) Cryptic species or inadequate taxonomy? Implementation of $2 \mathrm{~d}$ geometric morphometrics based on integumental organs as landmarks for delimitation and description of copepod taxa. Systematic Biology 65(2): 304-327.

Meleg IN, Zakšek V, Fišer C, Kelemen BS, Moldovan OT (2013) Can environment predict cryptic diversity? The case of niphargus inhabiting western carpathian groundwater. PLOS ONE 8(10): e76760.

\section{Glossary:}

Cryptic species: Different species, which are morphologically very similar or identical. Parallel evolution: Evolution of the same set of morphological traits from one ancestral set of traits. Convergent evolution: Evolution of the same set of morphological traits from different ancestral set of traits.

Stasis: Maintenance of morphological similarity during long time scales. Morphology: The form or structure of the external characters of an organism. 


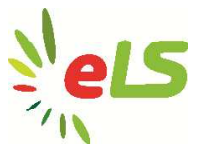

Figures and Tables:

Figure 1. Unifying conceptual framework based on Struck et al. (2018b). The x-axis represent time since divergence from the last common ancestor. Often genetic divergence is used as proxy for this. The $y$-axis represents the degree of phenotypic disparity. The dark blue area is the area of ongoing speciation and hence no species boundaries have been established yet (white circles). The light blue area indicates evolution between pairs of species (orange circles) as it is intuitively assumed. That is phenotypic disparity more or less increases linear with time. The orange area reflects cases (yellow circles) in which phenotypic evolution occurs at a much higher rate than anticipated such as. The green area represents cases (red circles) of significantly reduced phenotypic disparity given time as it is the case in the cryptic species.

Figure 2. Schematic representation of the Anopheles example for recent divergence (based on the results of Reidenbach et al 2012). The left panel exemplifies the recent divergence of the two cryptic species. In the middle the genomic inversion at chromosome 2 is shown and the right one lists the ecological differences observed between the two species.

Figure 3. Schematic representation of the Mastigias example of parallelism and convergence based on the results by Swift et al. (2016). The phylogenetic relationship between the two morphotypes (oceancic and lake; indicated by the two icons) is shown to the left. Swift et al. (2016) regarded the origin of the two lake morphotypes within the Chinese Sea as well as two within the Pacific Islands \& South Philippinean Seas as examples of parallelism as they originated from the same oceanic species. In contrast, they concluded that the other lake morphotypes to each other as well as to these previous ones evolved by on convergence as they originated from different oceanic species.

Figure 4. Schematic representation of the Cavernacmella example for stasis given the results of Wada et al. (2013). Wada et al. (2013) recognized a total five cryptic species as well as five morphologically distinct, non-cryptic species within them (indicated by the different forms and colours; icons are relative in size to each other). The occurrence of these ten species is indicated as well as their life-history and habitat (epigenean and cave-dwelling; indicated by the two icons).

Figure 5. Schematic representation how different biological disciplines can contribute to the research of cryptic species and thereby increase our understanding of macroecological, evolutionary and genomic patterns and processes.

\section{All 5 figures do not require permission as we generated them.}




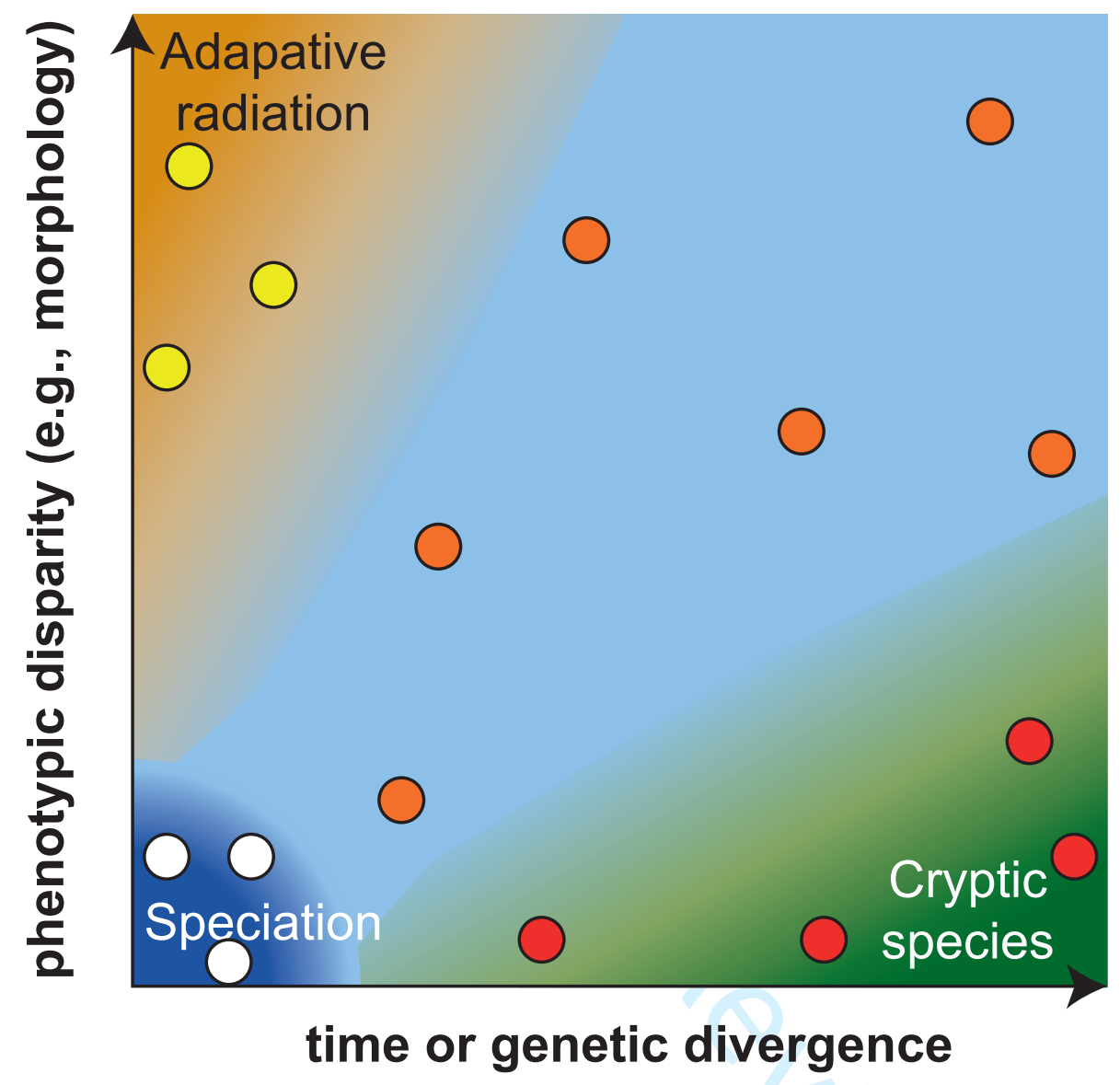



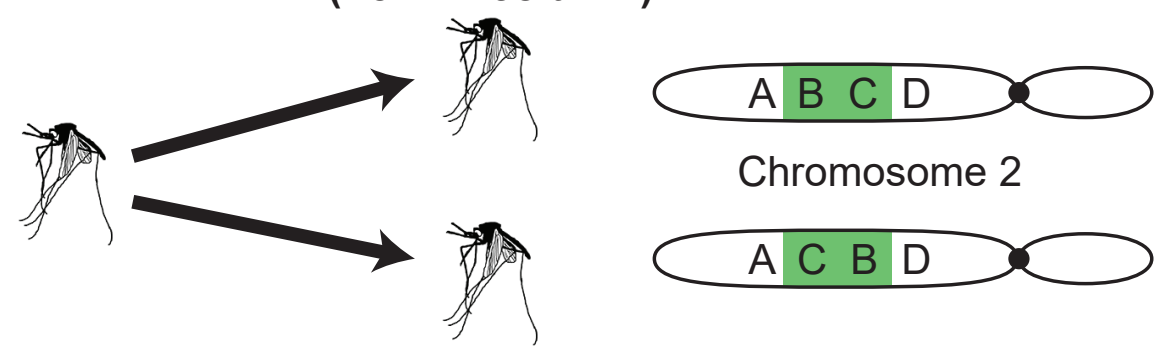

rice fields with high predator density polluted urban dumping grounds

*exchange

seasonal, temporary water bodies due to rainfall, predator free, unpolluted

$\mathrm{S}$ form

(still A. gambiae)

3,000 years

0

ago 
Parallelism Convergence

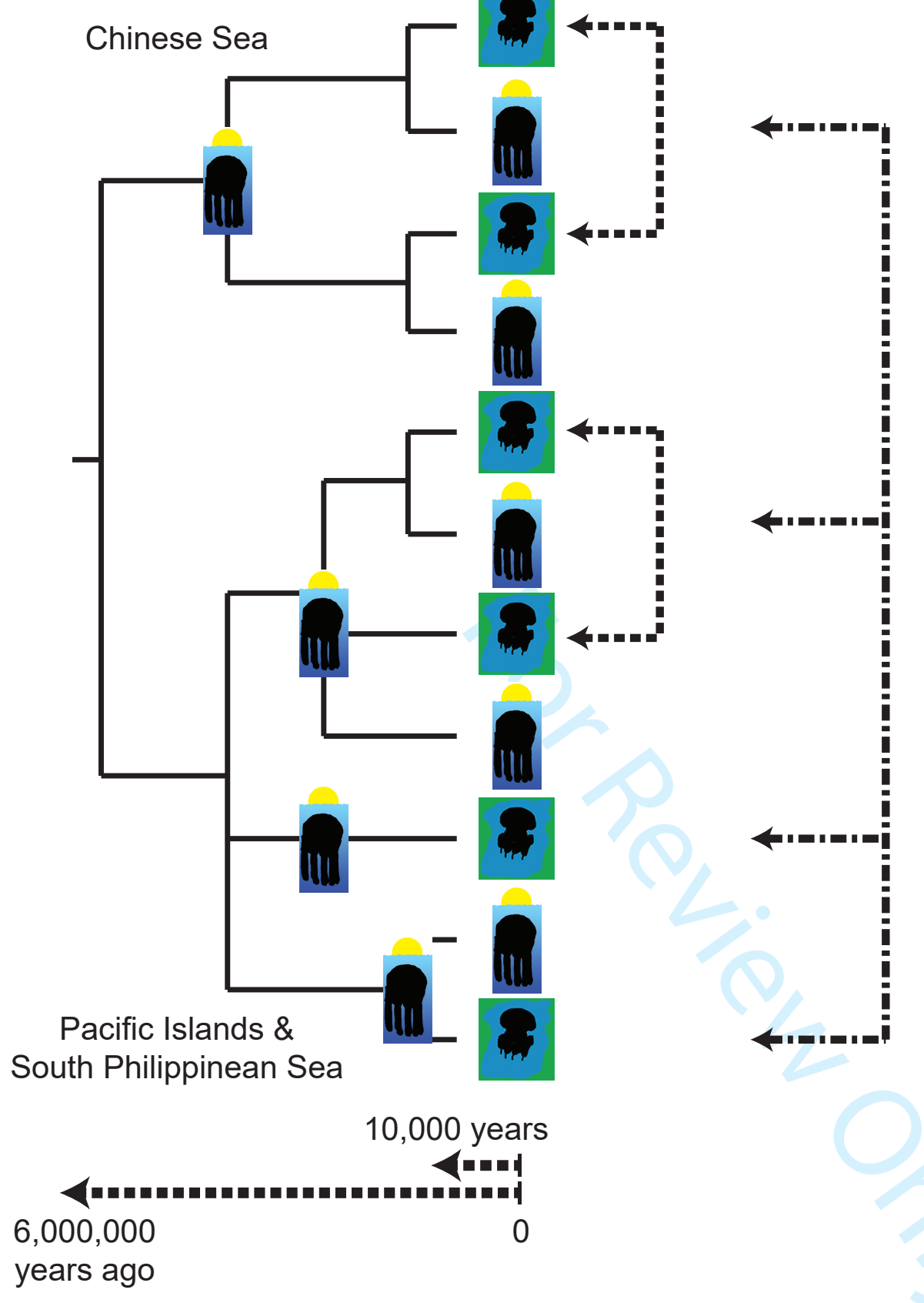




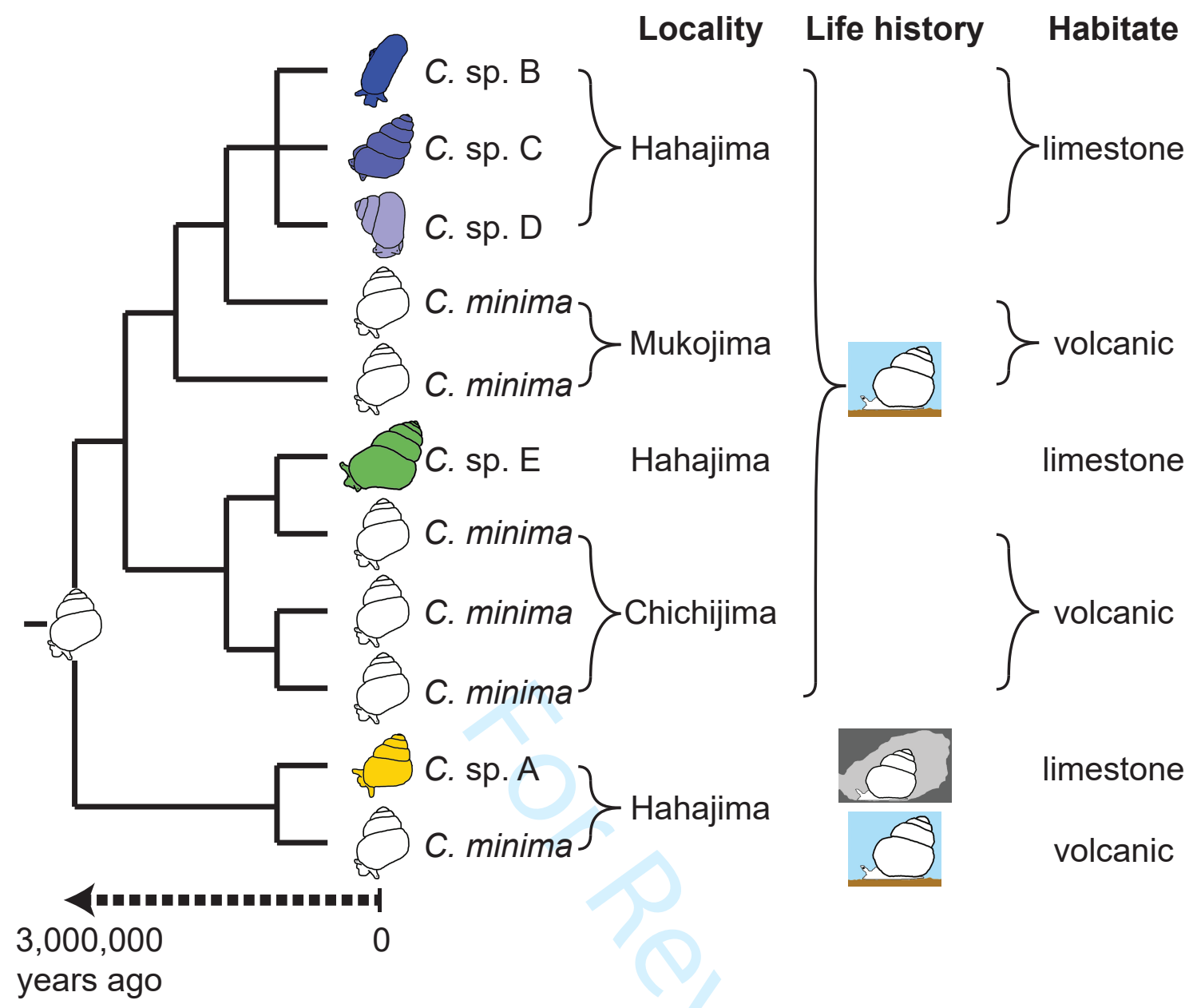




\section{Multidisciplinary research on cryptic species}

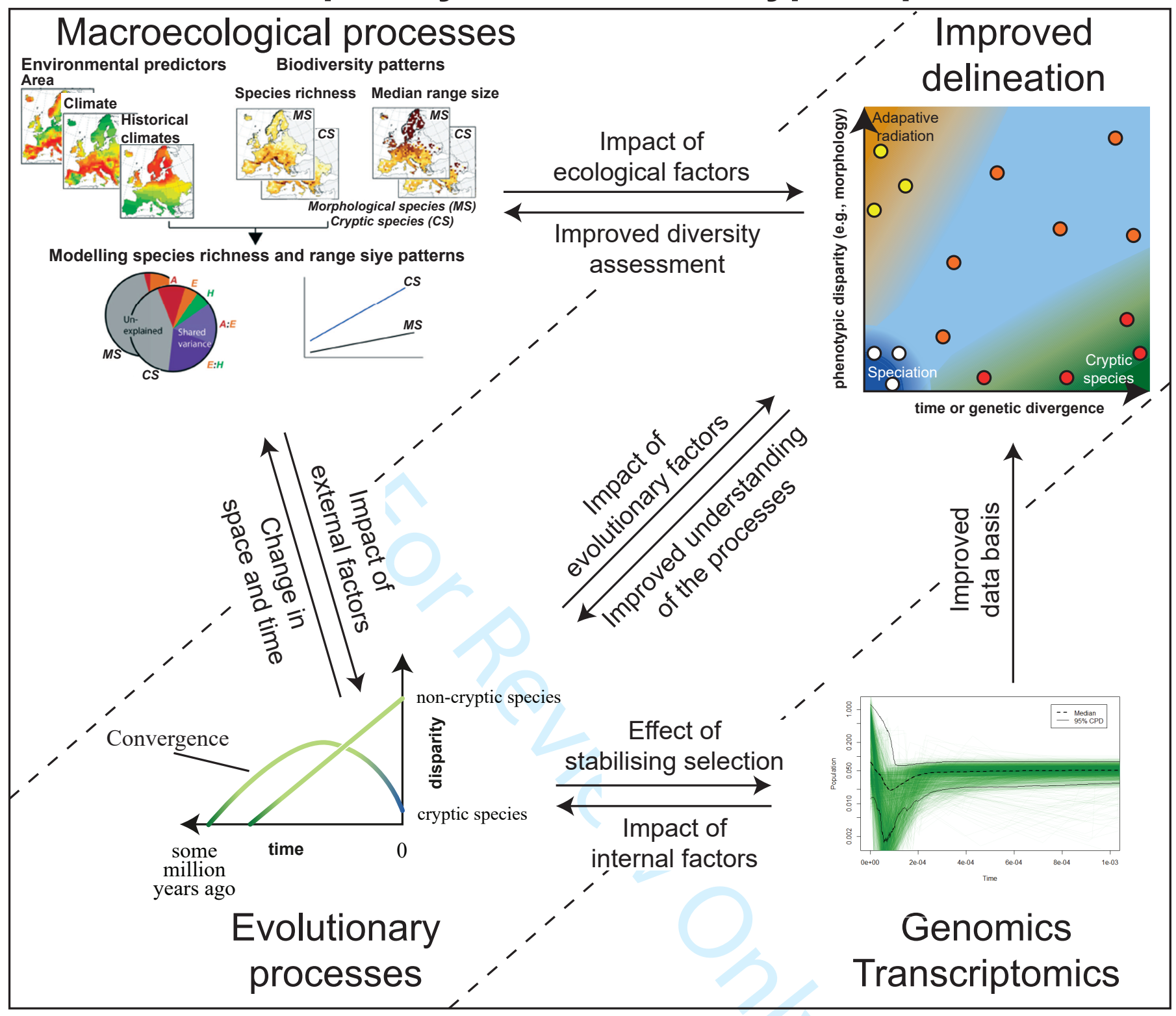

